\title{
A study of boundedness in probabilistic normed spaces
}

\author{
Bernardo Lafuerza-Guillén ${ }^{a}$, Carlo Sempi ${ }^{b, *}$, Gaoxun Zhang ${ }^{c}$ \\ a Departamento de Matemática Aplicada y Estadística, Universidad de Almería, Almería, Spain \\ bipartimento di Matematica "Ennio De Giorgi", Università del Salento, Lecce, Italy \\ 'Tian Fu College of Southwestern University of Finance and Economics Mianyang, Sichuan 621000, PR China
}

\section{A R T I C L E I N F O}

\section{Article history:}

Received 19 July 2009

Accepted 16 December 2009

\section{MSC:}

$54 \mathrm{E} 70$

46570

\section{Keywords:}

Probabilistic normed spaces

Boundedness

Archimedean triangle function

\begin{abstract}
A B S T R A C T
It was shown in Lafuerza-Guillén, Rodríguez-Lallena and Sempi (1999) [8] that uniform boundedness in a Šerstnev PN space $\left(V, v, \tau, \tau^{*}\right)$, (named boundedness in the present setting) of a subset $A \subset V$ with respect to the strong topology is equivalent to the fact that the probabilistic radius $R_{A}$ of $A$ is an element of $D^{+}$. Here we extend the equivalence just mentioned to a larger class of PN spaces, namely those PN spaces that are topological vector spaces (briefly TV spaces), but are not Šerstnev PN spaces.

We present a characterization of those PN spaces, whether they are TV spaces or not, in which the equivalence holds. Then, a characterization of the Archimedeanity of triangle functions $\tau^{*}$ of type $\tau_{T, L}$ is given. This work is a partial solution to a problem of comparing the concepts of distributional boundedness ( $D$-bounded in short) and that of boundedness in the sense of associated strong topology.
\end{abstract}

(C) 2010 Published by Elsevier Ltd

\section{Introduction}

Probabilistic Normed (briefly PN) spaces were introduced by Šerstnev in a series of papers [1-4]. Then a new definition was proposed by Alsina, Schweizer and Sklar [5]. This new definition revived the study of PN spaces. The properties of these spaces were studied by several authors; here we shall mention [6-9] (but see also the survey papers [10,11]).

We shall consider the space $\Delta$ of distribution functions (=d.f.'s); a d.f. is an increasing (in the weak sense) function to the subset $\Delta^{+}:=\{F \in[0,1]$ that is left-continuous and such that $F(-\infty)=0$ and $F(+\infty)=1$. The d.f.'s belonging proper distance distribution functions, be metrized in several for which $\lim _{x \rightarrow+\infty} F(x)=1$ will be needed. The space $\Delta$ can we metrized in several equivalent ways [12-14] in such a manner that the metric topology coincides with the topology of denoted by $d_{L}$ in [15]. The d.f.'s of constant rere, we assume that $\Delta$ is metrized by the Sibley metric $d_{S}$, which is the metric

$$
\varepsilon_{a}(t):= \begin{cases}0, & t \leq a, \\ 1, & t>a .\end{cases}
$$

For the reader's convenience, now we recall the definition of a PN space; the notation is essentially that fixed by the
ssical book by Schweizer and Sklar [15].

Definition 1.1. A probabilistic normed space is a quadruple $\left(V, \nu, \tau, \tau^{*}\right)$, where $V$ is a real linear space, $\tau$ and $\tau^{*}$ are continuous triangle functions and the mapping $v: V \rightarrow \Delta^{+}$satisfies, for all $p$ and $q$ in $V$, the conditions

\footnotetext{
* Corresponding author. Tel.: +39 0832351786; fax: +39 0832297410.

E-mail addresses: blafuerz@ual.es (B. Lafuerza-Guillén), carlo.sempi@unisalento.it (C. Sempi), zhanggaoxun002@163.com (G. Zhang). 
(N1) $v_{p}=\varepsilon_{0}$ if and only if $p=\theta(\theta$ is the null vector in $V)$;

(N2) $\forall p \in V \quad v_{-p}=v_{p}$;

(N3) $v_{p+q} \geq \tau\left(v_{p}, v_{q}\right)$;

(N4) $\forall \alpha \in[0,1] \quad v_{p} \leq \tau^{*}\left(v_{\alpha p}, v_{(1-\alpha) p}\right)$.

The function $\nu$ is called the probabilistic norm. If $\left(V, \nu, \tau, \tau^{*}\right)$ satisfies the condition, weaker than (N1),

$$
v_{t}=\varepsilon_{0},
$$

then it is called a Probabilistic Pseudo-Normed space (briefly, a PPN space). If $\tau=\tau_{T}$ and $\tau^{*}=\tau_{T^{*}}$ for some continuous $t$-norm $T$ and its $t$-conorm $T^{*}$ then $\left(V, v, \tau_{T}, \tau_{T^{*}}\right)$ is denoted by $(V, v, T)$ and is called a Menger PN space. A PN space is called a Šerstnev space if it satisfies (N1), (N3) and the following condition,

$(\check{S}) v_{\alpha p}(x)=v_{p}\left(\frac{x}{|\alpha|}\right)$ for every $\alpha \in \overline{\mathbb{R}} \backslash\{0\}$ and for every $x>0$.

From the point of view of topological vector spaces the most interesting PN spaces are those that are not Šerstnev spaces. In these cases vector addition is still continuous (provided the triangle function is determined by a continuous $t$-norm), while scalar multiplication, in general, is not continuous with respect to the strong topology (see [16]).

A set $A$ in the PN space $\left(V, v, \tau, \tau^{*}\right)$ is said to be $\mathscr{D}$-bounded if its probabilistic radius, $R_{A}$ belongs to $\mathscr{D}^{+}$. The probabilistic radius of $A$ is defined by

$$
R_{A}(x):= \begin{cases}\ell^{-} \inf \left\{v_{p}(x): p \in A\right\}, & x \in[0,+\infty[ \\ 1, & x=+\infty\end{cases}
$$

Of course, if $V$ is a normed space under the norm $\|\cdot\|$, then the set $A$ may be bounded when regarded as a subset of the normed space $(V,\|\cdot\|)$, but the two notions need not coincide (see [8]). The aim of this paper is to investigate the cases in which the notions of $\mathscr{D}$-boundedness and boundedness coincide. The papers [17-19] on the relationship between the two types of boundedness ought also to be kept in mind.

The notion of $t$-norm will be used in the sequel; its definition may be found in $[20,21,15]$. Briefly a $t$-norm is any binary operation $T$ on $[0,1]$ that is commutative, associative, increasing in each place and such that $T(a, 1)=a$ for every $a \in[0,1]$. A $t$-conorm $T^{*}$ is associated with every $t$-norm $T$; it is defined by $T^{*}(x, y):=1-T(1-x, 1-y)$

Important examples of $t$-norms and of their $t$-conorms are: $M(x, y)=\min \{x, y\}, \Pi(x, y)=x y$ and $M^{*}(x, y)=$ $\max \{x, y\}, \Pi^{*}(x, y)=x+y-x y$. We recall the following inequalities

$$
T \leq M<M^{*} \leq T^{*},
$$

which hold for every $t$-norm $T$; in particular

$$
W<\Pi<M<M^{*}<\Pi^{*}<W^{*} .
$$
Definition 1.2. A PN space $\left(V, \nu, \tau, \tau^{*}\right)$ will be said to be strict if $\nu(V) \subseteq \mathscr{D}^{+}$, or, equivalently, if $v_{p}$ belongs to $\mathfrak{D}^{+}$for every
$p \in V$.

Definition 1.3. A triangle function is a mapping $\tau$ from $\Delta^{+} \times \Delta^{+}$into $\Delta^{+}$such that, for all $F, G, H, K$ in $\Delta^{+}$,

(1) $\tau\left(F, \varepsilon_{0}\right)=F$,

(2) $\tau(F, G)=\tau(G, F)$,

(3) $\tau(F, G) \leq \tau(H, K)$ whenever $F \leq H, G \leq K$,

(4) $\tau(\tau(F, G), H)=\tau(F, \tau(G, H))$.

Particular and relevant triangle functions are the functions $\tau_{T}, \tau_{T^{*}}$ and those of the form $\Pi_{T}$ which, for every continuous $t$-norm $T$, and every $x>0$, are defined by

$$
\begin{aligned}
& \tau_{T}(F, G)(x)=\sup \{T(F(u), G(v)) \mid u+v=x\}, \\
& \tau_{T^{*}}(F, G)(x)=\inf \left\{T^{*}(F(u), G(v)) \mid u+v=x\right\}
\end{aligned}
$$

and

$$
\Pi_{T}(F, G)(x)=T(F(x), G(x)) .
$$

See $[22,23]$ for a recent study of triangle functions. Definition 1.4. A subset $A$ of a PN space which is a TV space is said to be bounded if for every $m \in \mathbf{N}$ there exists a $k \in \mathbf{N}$
such that

$$
A \subset k_{\mathcal{N}}(1 / m) .
$$


Definition 1.5. The subset $A$ is said to be totally bounded if for every $m \in \mathbf{N}$ there is a finite set $A_{1} \subseteq A$ such that

$$
A \subseteq \bigcup_{p \in A_{1}}\left(p+\mathcal{N}_{\theta}(1 / m)^{[k]}\right)
$$

Furthermore, whenever a metric space has the structure of a TV space, another type of boundedness is relevant. A subset $A$ of a TV space is topologically bounded if for every sequence $\left(\alpha_{n}\right) \subset \mathbb{R}$ with $\lim _{n \rightarrow+\infty}\left(\alpha_{n}\right)=0$ and for every sequence
$\left(p_{n}\right) \subset A$, one has $\lim _{n \rightarrow+\infty}\left(\alpha_{n} p_{n}\right)=\theta$.

Definition 1.6. Let $\mathscr{L}$ be the set of all binary operations $L$ on $\mathbb{R}^{+}$satisfying the following conditions:

(i) $\operatorname{Ran} L=\mathbb{R}^{+}$

(ii) $L$ is non-decreasing in each place;

(iii) $L$ is continuous on $\mathbb{R}^{+} \times \mathbb{R}^{+}$except, at most, in $(0, \infty)$ and $(\infty, 0)$.

Theorem 1.1. Let $T$ be a left-continuous $t$-norm, and let $L$ belong to $\mathscr{L}$ and satisfy the following conditions:

(a) L is commutative;

(b) L is associative;

(c) $u_{1}<u_{2}$ and $v_{1}<v_{2}$ imply $L\left(u_{1}, v_{1}\right)<L\left(u_{2}, v_{2}\right)$;

(d) $L(x, 0)=x$;

then the function $\tau_{T, L}$ is a triangle function.

Lemma 1.1. Let $T$ be a continuous $t$-norm and let L satisfy the condition of Theorem $1.1(a)$; then $\tau_{T, L}\left(\varepsilon_{a}, \varepsilon_{b}\right)=\varepsilon_{L(a, b)}$ for all a
and $b$ in $[0,+\infty[$.

Definition 1.7. A copula is a function $C:[0,1]^{2} \rightarrow[0,1]$ that satisfies the following conditions:

(C1) for every $t \in[0,1], C(0, t)=C(t, 0)=0$ and $C(1, t)=C(t, 1)=t$ :

(C2) $C$ is 2-increasing, i.e., for all $s, s^{\prime}, t$ and $t^{\prime}$ in $[0,1]$, with $s \leq s^{\prime}$ and $t \leq t^{\prime}$,

$$
C\left(s^{\prime}, t^{\prime}\right)-C\left(s^{\prime}, t\right)-C\left(s, t^{\prime}\right)+C(s, t) \geq 0 .
$$

It follows from Definition 1.7 that every copula $C$ is increasing in each place.

Moreover for any copula $C$ one has $W \leq C \leq M$.

Definition 1.8. Let $\varphi$ be a continuous, strictly decreasing function from $\mathbb{I}=[0,1]$ to $[0,+\infty]$ such that $\varphi(1)=0$. The pseudo-inverse of $\varphi$ is the function $\varphi^{[-1]}$ with $\operatorname{Dom} \varphi^{[-1]}=[0,+\infty]$ and $\operatorname{Ran} \varphi^{[-1]}=\mathbb{I}$ defined by

$$
\varphi^{[-1]}(t) ;= \begin{cases}\varphi^{-1}(t), & 0 \leq t \leq \varphi(0) \\ 0, & \varphi(0) \leq t \leq+\infty\end{cases}
$$

The following results are well known and can be found in [15] or in [24].

Lemma 1.2. Let $\varphi$ be a continuous, strictly decreasing function from $\mathbb{I}$ to $[0,+\infty]$ such that $\varphi(1)=0$, and let $\varphi^{[-1]}$ be the pseudo-inverse of $\varphi$ defined by (1.1). Let $C$ be the function from $\mathbb{I}^{2}$ to $\mathbb{I}$ defined by

$$
C(u, v)=\varphi^{[-1]}(\varphi(u)+\varphi(v)) .
$$

Then $C$ satisfies the boundary conditions (C1). Moreover $C$ is 2-increasing if, and only if, for all $v \in \mathbb{I}$,

$$
C\left(u_{2}, v\right)-C\left(u_{1}, v\right) \leq u_{2}-u_{1},
$$

whenever $u_{1} \leq u_{2}$,

Theorem 1.2. Let $\varphi$ be a continuous, strictly decreasing function from $\mathbb{I}$ to $[0,+\infty]$ such that $\varphi(1)=0$, and let $\varphi^{[-1]}$ be the pseudo-inverse of $\varphi$ defined by (1.1). Then the function $C$ from $\mathbb{I}^{2}$ to $\mathbb{I}$ defined by (1.2) is a copula if, and only if, $\varphi$ is convex.

Copulas of the form (1.2) are called Archimedean. An Archimedean copula is associative and a $t$-norm.

One owes the following result to Alsina, Schweizer and Sklar [16] 
Theorem 1.3. Every PN space $\left(V, \nu, \tau, \tau^{*}\right)$, when it is endowed with the strong topology induced by the probabilistic norm $\nu$, is a topological vector space if, and only if, for every $p \in V$ the map from $\mathbb{R}$ into $V$ defined by

$$
\lambda \mapsto \lambda p
$$

is continuous, i.e., for every $\eta>0$, there exists a number $\delta>0$ such that

$$
d_{S}\left(v_{\alpha^{\prime} p-\alpha p}, \varepsilon_{0}\right)<\eta
$$

whenever $\left|\alpha^{\prime}-\alpha\right|<\delta$, or, equivalently, such that $d_{S}\left(\nu_{\beta p}, \varepsilon_{0}\right)<\eta$ whenever $|\beta|<\delta$.

A triangle function $\tau$ is said to be Archimedean if $\varepsilon_{0}$ and $\varepsilon_{+\infty}$ are the only solutions in $\Delta^{+}$of the functional equation $\tau(F, F)=F$. Any such a solution is said to be an idempotent of the triangle function $\tau$; thus a triangle function $\tau$ is Archimedean if, and only if, $\varepsilon_{0}$ and $\varepsilon_{+\infty}$ are the only idempotents of $\tau$. Thus, for instance, the triangle functions $\tau_{W}$ and

It was proved in [16, Theorem 4], that, if the triangle function $\tau^{*}$ is Archimedean, then the mapping (1,4) is continuous in a Šerstnev PN space $\left(V, v, \tau, \tau^{*}\right)$, (named space $\left(V, v, \tau, \tau^{*}\right)$ is a TV space. It was shown in [8] that uniform boundedness topology is equivalent to the $\tau$, (named boundedness in the present setting) of a subset $A \subset V$ with respect to the strong to a larger class of PN spaces, namely those PN spaces radius $R_{A}$ of $A$ is an element of $D^{+}$. Here we extend this equivalence Šerstnev PN spaces.

Section 2 presents a characterization of those PN spaces, whether they are TV spaces or not, in which the equivalence holds. In Section 3, a characterization of the Archimedeanity of triangle functions $\tau^{*}$ of the type $\tau_{T, L}$ is given. This work is a partial solution to a problem of comparing the concepts of distributional boundedness ( $D$-bounded in short) and that of boundedness in the sense of associated strong topology.

\section{Main results (I)-A particular class of PN spaces} Definition 2.1. The PN space $\left(V, \nu, \tau, \tau^{*}\right)$ is said to satisfy the DI-condition ${ }^{1}$ if the probabilistic norm $v$ is such that, for all
$\alpha \in \mathbb{R} \backslash\{0\}, x \in \mathbb{R}$ and $p \in V$,

$$
v_{\alpha p}(x)=v_{p}(\varphi(\alpha, x)),
$$

where $\varphi: \mathbb{R} \times[0,+\infty[\rightarrow[0,+\infty[$ satisfies

$$
\lim _{x \rightarrow+\infty} \varphi(\alpha, x)=+\infty \text { and } \lim _{\alpha \rightarrow 0} \varphi(\alpha, x)=+\infty .
$$

Example 2.1. Let $(V,\|\cdot\|)$ be a normed space. For $\beta \in] 0,1\left[\right.$, define $v: V \rightarrow \Delta^{+}$by

$$
v_{p}(x):= \begin{cases}0, & x \leq 0, \\ \left(1-\beta \varepsilon_{0}(\|p\|)\right) \frac{\ln (1+x)}{\ln (1+x)+\|p\|}, & x \in] 0,+\infty[ \\ 1, & x=+\infty\end{cases}
$$

Below, we shall prove that $\left(V, \nu, \tau_{\Pi}, \tau_{M}\right)$

(1) is a PN space;

(2) is neither a Šerstnev space nor a TV space nor a strict PN space;

(3) satisfies the $D I$-condition, with

$$
\varphi(\alpha, x)=(x+1)^{1 /|\alpha|}-1 .
$$

(1) $\left(V, v, \tau_{\Pi}, \tau_{M}\right)$ is a PN space. It is easily verified that (N1) and (N2) hold. For all $p$ and $q$ in $V$, for all $s$ and $t$ in $R^{+}$, one has

$$
\frac{\|p+q\|}{\ln (1+s+t)} \leq \frac{\|p\|+\|q\|}{\ln (1+s+t)} \leq \frac{\|p\|}{\ln (1+s)}+\frac{\|q\|}{\ln (1+t)} \text {. }
$$

1 The acronym DI stands for the expression Double Infinity. 
Notice that the factor $\left(1-\beta \varepsilon_{0}(\|p\|)\right)$ equals 1 if, and only if, $p=\theta$; otherwise, it equals $1-\beta$. Then, if both $p$ and $q$ differ
from $\theta$,

$$
\begin{aligned}
v_{p+q}(s+t) & =(1-\beta) \frac{\ln (1+s+t)}{\ln (1+s+t)+\|p+q\|}=(1-\beta) \frac{1}{1+\frac{\|p+q\|}{\ln (1+s+t)}} \\
& \geq(1-\beta) \frac{1}{1+\frac{\|p\|}{\ln (1+s)}+\frac{\|q\|}{\ln (1+t)}} \\
& \geq(1-\beta)^{2} \frac{1}{1+\frac{\|p\|}{\ln (1+s)}+\frac{\|q\|}{\ln (1+t)}+\frac{\|p\|\|q\|}{\ln (1+s) \ln (1+t)}} \\
& =\frac{(1-\beta)}{1+\frac{\|p\|}{\ln (1+s)}} \cdot \frac{(1-\beta)}{1+\frac{\|q\|}{\ln (1+t)}} \\
& =(1-\beta) \frac{\ln (1+s)}{\ln (1+s)+\|p\|} \cdot(1-\beta) \frac{\ln (1+t)}{\ln (1+t)+\|q\|}=v_{p}(s) \cdot v_{q}(t) .
\end{aligned}
$$

As a consequence (N3) holds, i.e., for all $p$ and $q$ in $V$, and for every $x \in R^{+}$,

$$
v_{p+q}(x) \geq \tau_{\pi}\left(v_{p}, v_{q}\right)(x) .
$$

This latter inequality is trivially true if at least one of $p$ and $q$ equals $\theta$.

For all $s$ and $t$ in $R^{+}$, one has

$$
\begin{aligned}
1-\frac{\ln (1+s)}{\ln (1+s+t)} & =\frac{\ln (1+s+t)-\ln (1+s)}{\ln (1+s+t)}=\frac{\ln \frac{s+t+1}{s+1}}{\ln (1+s+t)} \\
& =\frac{\ln \left(1+\frac{t}{s+1}\right)}{\ln (1+s+t)} \leq \frac{\ln (1+t)}{\ln (1+s+t)} .
\end{aligned}
$$

Therefore, because of Lemma 2 in [7],

$$
\nu\left(1-\frac{\ln (1+s)}{\ln (1+s+t)}\right) p \geq \nu \frac{\ln (1+t)}{\ln (1+s+t)} p^{.}
$$

For all $\alpha \in] 0,1\left[\right.$ and $x \in R^{+}$, there exist $s_{0}, t_{0}$ in $R^{+}$such that $s_{0}+t_{0}=x$ and

$$
\alpha=\frac{\ln \left(1+s_{0}\right)}{\ln \left(1+s_{0}+t_{0}\right)} ;
$$

in fact $s_{0}=(1+x)^{\alpha}-1$. Whence, if $p \neq \theta$.

$$
\begin{aligned}
\tau_{M}\left(v_{\alpha p}, v_{(1-\alpha) p}\right)(x) & =\sup _{s+t=x} \min \left(v_{\alpha p}(s), v_{(1-\alpha) p}(t)\right) \\
& =\sup _{s+t=x} \min \left(v_{\left(\frac{\ln \left(1+s_{0}\right)}{\ln \left(1+s_{0}+t_{0}\right)}\right) p}(s), v\left(1-\frac{\ln \left(1+s_{0}\right)}{\ln \left(1+s_{0}+t_{0}\right)}\right) p\right. \\
& (t)) \\
& \geq \min \left(v\left(\frac{\ln \left(1+s_{0}\right)}{\ln \left(1+s_{0}+t_{0}\right)}\right) p\right. \\
& \left(s_{0}\right), v\left(1-\frac{\ln \left(1+s_{0}\right)}{\ln \left(1+s_{0}+t_{0}\right)}\right) p \\
& \left.\left(t_{0}\right)\right) \\
& \geq \min \left(v_{\left(\frac{\ln \left(1+s_{0}\right)}{\ln \left(1+s_{0}+t_{0}\right)}\right) p}\left(s_{0}\right), v\left(\frac{\ln \left(t_{0}+1\right)}{\ln \left(1+s_{0}+t_{0}\right)}\right) p\right. \\
& \left.\left(t_{0}\right)\right) \\
& =\min \left(\frac{(1-\beta) \ln \left(1+s_{0}+t_{0}\right)}{\ln \left(1+s_{0}+t_{0}\right)+\|p\|}, \frac{(1-\beta) \ln \left(1+s_{0}+t_{0}\right)}{\ln \left(1+s_{0}+t_{0}\right)+\|p\|}\right) \\
& =\min \left(v_{p}(x), v_{p}(x)\right)=v_{p}(x) .
\end{aligned}
$$

Therefore (N4) holds for $p \neq \theta$; since (N4) is obvious for $p=\theta$, it holds for every $p \in V$ and for every $\alpha \in] 0,1[$.
For $\alpha=0$ or $\alpha=1$, (N4) obviously holds, i.e.

$$
v_{p}=\tau_{M}\left(v_{\alpha p}, v_{(1-\alpha) p}\right) \text {. }
$$

(2) $\left(V, v, \tau_{\Pi}, \tau_{M}\right)$ is neither a Šerstnev space nor a TV space nor a strict PN space. A straightforward calculation shows that $v_{\alpha p}(x) \neq v_{p}(x /|\alpha|)$ so that $\left(V, \nu, \tau_{\pi}, \tau_{M}\right)$ is not a Šerstnev space.

We recall that a sufficient condition for a PN space $\left(V, \nu, \tau, \tau^{*}\right)$ to be a TV space is that $\tau^{*}$ be an Archimedean triangle function. Now, $\tau_{M}$ is not Archimedean so that all one needs to prove is that the scalar multiplication $\lambda \mapsto \lambda p$ is not 
continuous. For every sequence $\left(\lambda_{n}\right)$ of real numbers that converges to 0 as $n$ tends to $+\infty$, and for every $p$ in $V$ one has $\lambda_{n} p \nrightarrow \theta$ in the strong topology of $V$. Now

$$
\lim _{n \rightarrow \infty} v_{\lambda_{n} p}(x)=1-\beta<1
$$

or equivalently,

$$
\lim _{n \rightarrow \infty} v_{\lambda_{n} p} \neq \varepsilon_{0} .
$$

Also since $\lim _{x \rightarrow \infty} \nu_{p}(x)=1-\beta<1$, the distance d.f. $v_{p}$ is in $\Delta^{+} \backslash D^{+}$, so that $\left(V, v, \tau_{\pi}, \tau_{M}\right)$ is not a strict PN space.

(3) $\left(V, \nu, \tau_{\Pi}, \tau_{M}\right)$ satisfies the DI-condition. For all $x \in \mathbb{R}^{+}, \lambda \in \mathbb{R} \backslash\{0\}$ and $p \in V$, one has, for $p \neq \theta$,

$$
\begin{aligned}
v_{\lambda p}(x) & =\frac{(1-\beta) \ln (1+x)}{\ln (1+x)+|\lambda|\|p\|}=\frac{(1-\beta) \frac{1}{|\lambda|} \ln (1+x)}{\frac{1}{|\lambda|} \ln (1+x)+\|p\|} \\
& =\frac{(1-\beta) \ln (1+x)^{\frac{1}{\lambda \mid}}}{\ln (1+x)^{\frac{1}{|\lambda|}}+\|p\|}=v_{p}\left((1+x)^{1 /|\lambda|}-1\right) .
\end{aligned}
$$

Let

$$
\varphi(\lambda, x)=(1+x)^{1 /|\lambda|}-1 .
$$

It is easily checked that $(\lambda, x) \mapsto \varphi(\lambda, x)$ satisfies

$$
\lim _{x \rightarrow+\infty} \varphi(\lambda, x)=+\infty \text { and } \lim _{\lambda \rightarrow 0} \varphi(\lambda, x)=+\infty .
$$

Example 2.2. Let $\left(V, v, \tau, \tau^{*}\right)$ be an $\alpha$-Šerstnev space (see [6]). It is easy to show that $\left(V, v, \tau, \tau^{*}\right)$ satisfies the $D I$-condition,
where

$$
\varphi(\lambda, x)=\frac{x}{|\lambda|^{\alpha}}
$$

Example 2.3. The quadruple $\left(V, v, \Pi_{\Pi}, \Pi_{M}\right)$ where $V$ is a normed linear space and $v$, the probabilistic norm, is a map $v: V \rightarrow \Delta^{+}$defined via $v_{p}(x):=\mathrm{e}^{-\|p\| / x}$ for $x>0$, is a PN space as that of Example 2.2 with $\alpha=1$.

Notice that this probabilistic norm is an increasing function of $x$ and a decreasing function of $\|p\|$.

Property (N3) and (N2) are obvious.

Property (N3) is obviously true since

$$
\mathrm{e}^{-\|p+q\| / x} \geq \mathrm{e}^{-\|p\| / x} \cdot \mathrm{e}^{-\|q\| / x} .
$$

As for property (N4), since $\|p\| \geq \lambda\|p\|$ for every $p \in V$ and for every $\lambda \in[0,1]$, one has for every $\alpha \in] 0,1[$

$$
\mathrm{e}^{-\|p\| / x} \leq \min \left\{\mathrm{e}^{-\|\alpha p\| / x}, \mathrm{e}^{-\|(1-\alpha) p\| / x}\right\} .
$$

Moreover, this is a Šerstnev PN space, as is immediately seen.

It is also a topological vector space. As a matter of fact, for every $p \neq \theta$, and for every arbitrary sequence $\left(\alpha_{n}\right)$ with $\alpha_{n} \neq 0$, $(n \in \mathbf{N})$ such that $\alpha_{n} \rightarrow 0$ as $n$ tends to $+\infty$, one has

$$
\nu_{\alpha_{n} p}(x)=\mathrm{e}^{-\left|\alpha_{n}\right|\|p\| / x} \underset{n \rightarrow+\infty}{\longrightarrow} 1 \text {. }
$$

Moreover there is a neighbourhood of the origin $\theta$ that is topologically bounded: For every sequence $\left(\alpha_{n}\right)$ with $\alpha_{n} \neq 0$, $(n \in \mathbf{N})$ such that $\alpha_{n} \rightarrow 0$ as $n$ tends to $+\infty$, and for every sequence $\left(p_{n}\right)$ of elements of $\mathcal{N}_{\theta}(t)$ one has

$$
\nu_{\alpha_{n} p_{n}}(t)=\mathrm{e}^{-\left|\alpha_{n}\right|\left\|p_{n}\right\| / t} \underset{n \rightarrow+\infty}{\longrightarrow} 1 \text {. }
$$

Theorem 2.1. Let $\left(V, v, \tau, \tau^{*}\right)$ be a PN space that satisfies the DI-condition. Then for a subset $A \subset V$ the following statements
are equivalent:
(a) $A$ is D-bounded.
(b) $A$ is bounded, namely, for every $n \in \mathbf{N}$ and for every $p \in A$, there is $k \in \mathbf{N}$ such that $v_{p / k}(1 / n)>1-1 / n$.
(c) $A$ is topologically bounded. 
Proof. (a) $\Longrightarrow$ (b). Let $A$ any $D$-bounded subset of $V$. By definition of $D$-boundedness, the probabilistic radius $R_{A}$ of $A$ (see [8]) is a distance d.f. such that $\lim _{x \rightarrow+\infty} R_{A}(x)=1$, Therefore, for every $n \in \mathbf{N}$, there exists $x_{n}>0$ such that $R_{A}\left(x_{n}\right)>1-1 / n$. Thus, for every $p \in A$,

$$
v_{p}\left(x_{n}\right) \geq R_{A}\left(x_{n}\right)>1-1 / n .
$$

Since, in view of the $D I$-condition,

$$
\lim _{\lambda \rightarrow 0} \varphi(\lambda, 1 / n)=+\infty,
$$

for every $n \in \mathbf{N}$, there exists $\lambda^{\prime} \in \mathbb{R}$ such that $\varphi\left(\lambda^{\prime}, 1 / n\right) \geq x_{n}$. Therefore,

$$
v_{\lambda^{\prime} p}(1 / n)=v_{p}\left(\varphi\left(\lambda^{\prime}, 1 / n\right)\right) \geq v_{p}\left(x_{n}\right) \geq R_{A}\left(x_{n}\right)>1-1 / n .
$$

As a consequence letting $k=\left[1 / \lambda^{\prime}\right]$, where $[t]$ denotes the integral part of $t$, one has

$$
\nu_{p / k}(1 / n)>1-1 / n
$$

namely, $A$ is bounded.

(b) $\Longrightarrow$ (a) Let $A$ be a bounded subset of $V$ and consider the neighbourhood of $\theta, N_{\theta}(1 / n)$. Then there exists $\lambda_{0} \in \mathbb{R}$ such that for every $p \in V, p=\lambda_{0} q$ for some $q \in N_{0}(1 / n)$.

Because of the $D I$-condition, $\lim _{x \rightarrow+\infty} \varphi\left(\lambda_{0}, x\right)=+\infty$, for every $n \in \mathbf{N}$; then, there exists $x_{0}>0$ such that, $\varphi\left(\lambda_{0}, x_{0}\right) \geq 1$. Then, for $x \geq x_{0}$

$$
v_{p}(x)=v_{\lambda_{0} q}(x) \geq v_{\lambda_{0} q}\left(x_{0}\right)=v_{q}\left(\varphi\left(\lambda_{0}, x_{0}\right)\right) \geq v_{q}(1) \geq v_{q}(1 / n)>1-1 / n,
$$

so that

$$
R_{A}(x) \geq 1-\frac{1}{n}
$$

i.e., $R_{A}$ is in $D^{+}$.

$($ a) $\Longrightarrow$ (c). Let $A$ any $D$-bounded subset of $V$. One has, as above,

$$
v_{\alpha_{n} p_{n}}\left(x_{n}\right)=v_{p_{n}}\left(\varphi\left(\alpha_{n}, x_{n}\right)\right) \geq R_{A}\left(x_{n}\right)>1-\frac{1}{n},
$$

which implies

$$
\alpha_{n} p_{n} \underset{n \rightarrow+\infty}{\longrightarrow} \theta .
$$

(c) $\Longrightarrow$ (a). Let $A$ be a subset of $V$ which is not $D$-bounded. Then

$$
\lim _{x \rightarrow+\infty} R_{A}(x)=\gamma<1 .
$$

By definition of $R_{A}$, for every $n \in \mathbf{N}$ there is $p_{n} \in A$ such that, for every $x>0$,

$$
\nu_{p_{n}}(x)<\frac{1+\gamma}{2}<1
$$

Then for every $x>0$,

$$
v_{\alpha_{n} p_{n}}(x)=v_{p_{n}}\left(\varphi\left(\alpha_{n}, x\right)\right)<\frac{1+\gamma}{2}<1,
$$

which shows that $v_{\alpha_{n} p_{n}}$ does not tend to $\varepsilon_{0}$, even if it has a weak limit, viz., $\left(\alpha_{n} p_{n}\right)$ does not tend to $\theta$ in the strong topology; in other words, $A$ is not topologically bounded.

The next example exhibits a PN space $\left(V, v, \tau, \tau^{*}\right)$ that is a TV space but in which the triangle function $\tau^{*}$ is not Archimedean.

Example 2.4. Let $\left(V, v, \tau, \tau^{*}\right)$ be a PN space in which the triangle function $\tau^{*}$ is not Archimedean. Let $f$ and $g$ be functions satisfying the following conditions:

(i) $f:[0,+\infty] \rightarrow \mathbb{I}$ is continuous, decreasing and $f(0)=1$;

(ii) $g: \mathbb{R}^{+} \times \mathbb{R}^{+} \rightarrow \mathbb{I}$ is continuous in either place, increasing in the first place and decreasing in the second place with $g(x, 0)=1$.

Then the quadruple $\left(V, v, \tau, \tau^{*}\right)$ where the probabilistic norm $v$ is defined via

$$
\nu_{p}(x):=f(\|p\|) \cdot g(x,\|p\|),
$$

is a TV space. 
Notice that every PN space $\left(V, \nu, \tau, \tau^{*}\right)$ in which $\tau^{*}=\tau_{M}$ and such that $\nu(V) \subseteq D^{+}$is a TV space since $\tau_{M}$ is Archimedean on the subset $D^{+}$of $\Delta^{+}$.

The condition $v(V) \subseteq \mathcal{D}^{+}$is not necessary to obtain a TV space as shown in [25, Theorem 9] and in the following example.

Example 2.5. With the same assumptions of the Example 2.4 one has that the quadruple $\left(V, \nu, \Pi_{\Pi}, \Pi_{M}\right)$ where

$$
\nu_{p}(x)=\frac{1}{1+\|p\|} \cdot \frac{x}{x+\|p\|}
$$

is a PN space that is a TV space, which is not Šerstnev and is not strict.

Example 2.6. The quadruple $\left(V, v, \Pi_{\Pi}, \Pi_{M}\right)$ where the probabilistic norm $v$ is defined via

$$
\nu_{p}(x):=\exp \left(-\frac{\|p\| x}{\|p\| x+1}\right),
$$

is a PN space that is neither Šerstnev nor strict, but it is a TV space.

Example 2.7 ([16]). For $p \in \mathbb{R}$ define $v$ by setting $v_{0}=\varepsilon_{0}$, and

$$
\nu_{p}:=\frac{1}{\|p\|+2} \varepsilon_{0}+\frac{\|p\|+1}{\|p\|+2} \varepsilon_{\infty},
$$

for $p \neq 0$, it is straightforward to show that $\left(\mathbb{R}, v, \tau_{W}, \tau_{M}\right)$ is a PN space, and that, for any $p \neq 0$ and any sequence $\left(\alpha_{n}\right)$ converging to 0 , the sequence $\left(\nu_{\alpha_{n} p}\right)$ converges weakly, not to $\varepsilon_{0}$, but to $\left(\varepsilon_{0}+\varepsilon_{\infty}\right) / 2$. Thus $v_{\alpha p}$ is not continuous in its first place, namely, scalar multiplication is not a continuous mapping from $\mathbb{R}$ into $V$. Thus $\left(\mathbb{R}, v, \tau_{W}, \tau_{M}\right)$ is not a TV space.

Example 2.8. Let $(V,\|\cdot\|)$ be a normed space and, for $\alpha \in] 0,1\left[\right.$, let $v: V \rightarrow \Delta^{+}$be given by

$$
\nu_{p}(x)= \begin{cases}0, \quad x \leq 0, & \\ \frac{\ln (1+x)}{\ln (1+x)+\|p\|}, & x \in] 0,+\infty[,\|p\|<1 \\ \frac{a \ln (1+x)}{\ln (1+x)+\|p\|}, & x \in] 0,+\infty[,\|p\| \geq 1, \\ 1, & x=+\infty .\end{cases}
$$

Then

(1) $\left(V, v, \tau_{\pi}, \tau_{M}\right)$ is a PN space satisfying the $D I$-condition with

$$
\varphi(\lambda, x)=(1+x)^{\frac{1}{\|\lambda\|}}-1
$$

(2) $\left(V, v, \tau_{\pi}, \tau_{M}\right)$ is a TV PN space;

(3) the subset $A=\{p:\|p\|<1\}$ is both $D$-bounded and bounded.

Only property (2) needs to be checked. For every sequence $\left(\lambda_{n}\right)$ of real numbers that converges to 0 as $n$ tends to $+\infty$, and for every $p \in V$, one has $\lambda_{n} p \rightarrow \theta$ in the strong topology of $V ;$ in fact, for every $x \in \mathbb{R}^{+}, \lim _{n \rightarrow \infty} \nu_{\lambda_{n} p}(x)=1$, namely, $\lim _{n \rightarrow \infty} \nu_{\lambda_{n} p}=\varepsilon_{0}$.

\section{II-A class of PN spaces that are TV spaces}

Theorem 1.1 implies that $L \geq \max$; one also knows that $\tau_{T, L} \leq \Pi_{T}$.

Lemma 3.1. Let $L \in \mathcal{L}$ satisfy the assumptions of Theorem 1.1 ; then $L=\max$, if, and only if, $L(a, a)=$ a for every $a \in] 0,+\infty[$.

The following theorem studies whether certain classes of PN spaces are TV spaces.

Theorem 3.1. Let $T$ be an Archimedean $t$-norm and assume that $L \in \mathcal{L}$ satisfies the assumptions of Theorem 1.1, then the following statements are equivalent:

(a) $\tau_{T, L}$ is a triangle function having no nontrivial idempotents in $\Delta^{+}$;

(b) L satisfies the condition $L(x, x)>x$ for every $x \in] 0,+\infty[$. 
Proof. Recall that $\tau_{T, L}$ is a continuous triangle function (see [15, Theorems 7.2.4 and 7.2.8]).

$(\mathrm{a}) \Longrightarrow$ (b) A continuous triangle function with no nontrivial idempotents in $\Delta^{+}$is Archimedean (see [15, Lemma 14.1.3]). Then $\tau_{T, L}$ is an Archimedean triangle function, and, for every function $F \notin\left\{\varepsilon_{0}, \varepsilon_{\infty}\right\}$, one has $\tau_{T, L}(F, F) \neq F$. By Lemma 1.1, $\varepsilon_{x} \neq \tau_{T, L}\left(\varepsilon_{x}, \varepsilon_{x}\right)=\varepsilon_{L(x, x)}$ for every $\left.x \in\right] 0,+\infty$ [. Therefore $L(x, x) \neq x$ and from Lemma 3.1 one has $L(x, x)>x$ for every $x \in] 0,+\infty[$, which is the assertion.

(b) $\Longrightarrow$ (a) Let $F \in \Delta^{+}$be different from $\varepsilon_{a}$ for every $a \in[0,+\infty]$. Then there exists $\left.x_{0} \in\right] 0,+\infty$ [ such that $F\left(x_{0}\right)$ is in ]0, $1[$. Since $T$ is Archimedean, the following holds

$$
\tau_{T, L}(F, F)\left(x_{0}\right)=\sup \left\{T(F(u), F(v)): L(u, v)=x_{0}\right\} \leq T\left(F\left(x_{0}\right), F\left(x_{0}\right)\right)<F\left(x_{0}\right) .
$$

Therefore $F$ is not an idempotent element of $\tau_{T, L}$. Now let $x$ be in ]0, $+\infty$ [. By Lemma 1.1,

$$
\tau_{T, L}\left(\varepsilon_{x}, \varepsilon_{x}\right)=\varepsilon_{L(x, x)} \neq \varepsilon_{x},
$$

whence $\tau_{T, L}$ has no nontrivial idempotents elements. This concludes the proof.

Example 3.1. If $C$ is an Archimedean copula, then the triangle function $\tau_{C, L}$ where $L \in \mathscr{L}$ satisfies the assumptions of Theorem 1.1 is Archimedean.

Theorem 3.2. Let $T_{1}$ and $T_{2}$ be t-norms such that $T_{1} \leq T_{2}$; then $\tau_{T_{1}, L}$ and $\tau_{T_{2}, L}$ have the same idempotents.

Proof. If $F$ is in $\Delta^{+}$and $\tau_{T_{1}, L}$ admits $F$ as idempotent, then

$$
F=\tau_{T_{1}, L}(F, F) \leq \tau_{T_{2}, L}(F, F) \leq F,
$$

so that $\tau_{T_{2}, L}(F, F)=F$.

Corollary 3.1. Let $T$ be a $t$-norm such that $T \leq \Pi$, then the family of all $\mathrm{PN}$ spaces of the type $\left(V, \nu, \tau_{T, L}, \tau_{\Pi, L}\right)$ are $T V$ spaces.

Corollary 3.2. Let $T$ be a $t$-norm such that $T \leq W$, then the family of all PN spaces of the type $\left(V, \nu, \tau_{T, L}, \tau_{W, L}\right)$ are $T V$ spaces.

Theorem 3.3. Let $T$ and $C$ be a continuous t-norm and an Archimedean copula, respectively; if, for every $(u, v) \in[0,1]^{2}$, $T(u, v) \leq C(u, v)$, then every PN space of the form $\left(V, v, \tau_{T, L}, \tau_{C, L}\right)$ is a TV space.

The above result provides many examples of Archimedean triangle functions. It suffices to take

$$
L=K_{\alpha}(x, y)=\left(x^{\alpha}+y^{\alpha}\right)^{1 / \alpha} \quad\left(x, y \in \mathbb{R}^{+} ; \alpha \geq 1\right) .
$$

\section{Acknowledgement}

The first author was supported by grants from Ministerio de Ciencia e Innovación (MTM2006-12218).

\section{References}

[1] A.N. Šerstnev, Random normed spaces: Problems of completeness, Kazan Gos. Univ. Učen. Zap. 122 (1962) 3-20.

[2] A.N. Serstnev, On the notion of a random normed space, Dokl. Akad. Nauk. SSSR 149 (2) (1963) 280-283; English transl. Soviet Math. Doklady 4 (1963) 388-390.

[3] A.N. Šerstnev, Best approximation problems in random normed spaces, Dokl. Akad. Nauk. SSSR 149 (3) (1963) 539-542.

[4] A.N. Šerstnev, On a probabilistic generalization of a metric spaces, Kazan Gos. Univ. Učen. Zap. 124 (1964) 3-11.

[5] C. Alsina, B. Schweizer, A. Sklar, On the definition of a probabilistic normed space, Aequationes Math. 46 (1993) 91-98.

[6] B. Lafuerza-Guillén, J.A. Rodríguez-Lallena, C. Sempi, Some classes of probabiistic normed spaces, Rend. Mat. 17 (1997) $237-252$.

[7] B. Lafuerza-Guillén, J.A. Rodríguez-Lallena, C. Sempi, Completion of probabilistic normed spaces, Internat. J. Math. \& Math. Sci. 18 (1995) 649-652.

[8] B. Lafuerza-Guillén, J.A. Rodríguez-Lallena, C. Sempi, A study of boundedness in probabilistic normed spaces, J. Math. Anal. Appl. 232 (1999) $183-196$.

[9] B. Lafuerza-Guillén, J.A. Rodríguez-Lallena, C. Sempi, Probabilistic norms for linear operators, J. Math. Anal. Appl. 220 (1998) $462-476$.

[10] B. Lafuerza-Guillén, Latest results on probabilistic normed spaces, Int. J. Math. Anal. 1 (2006) 269-309.

[11] C. Sempi, A short and partial history of probabilistic normed spaces, Mediterr. J. Math. 3 (2006) 283-300. doi:10.1007/s00009-006-0078-6.

[12] B. Schweizer, Multiplication on the space of probability distribution functions, Aequationes Math. 12 (1975) 156-183.

[13] D.A. Sibley, A metric for weak convergence of distribution functions, Rocky Mountain J. Math. 1 (1971) 427-430.

[14] M.D. Taylor, New metrics for weak convergence of distribution, functions, Stochastica 9(1985) 5-17.

[15] B. Schweizer, A. Sklar, Probabilistic Metric Spaces, Elsevier, New York, 1983; 2nd ed. Dover, Mineola, NY, 2005.

[16] C. Alsina, B. Schweizer, A. Sklar, Continuity property of probabilistic norms, J. Math. Anal. Appl. 208 (1997) 446-452.

[17] U. Hoehle, Probabilistic metrization of generalized topologies, Bull. Acad. Polon. Sci. Série des Sciences Math., Astr. et Phys. 25 (1977) $493-498$

[18] E.O. Thorp, Generalized topologies for statistical metric spaces, Fund. Math. 51 (1962) 9-21.

[19] B. Lafuerza-Guillén, J.L. Rodríguez, Translation-invariant generalized topologies induced by probabilistic norms, Note Mat. (in press).

[20] C. Alsina, M.J. Frank, B. Schweizer, Associative Functions. Triangular Norms and Copulas, World Scientific, Singapore, 2006.

[21] E.P. Klement, R. Mesiar, E. Pap, Triangular Norms, Kluwer, Dordrecht, 2000

[22] S. Saminger-Platz, C. Sempi, A primer on triangle functions. I, Aequationes Math. 76 (2008) 201-240.

[23] S. Saminger-Platz, C. Sempi, A primer on triangle functions. II, Aequationes Math. (in press).

[24] R.B. Nelsen, An Introduction to Copulas, 2nd ed., Springer, New York, 2006

[25] B. Lafuerza-Guillén, J.A. Rodríguez-Lallena, C. Sempi, Normability of probabilistic normed spaces, Note Mat. (in press). 